\title{
O uso indiscriminado do Ritalina para o melhoramento no desempenho acadêmico
}

\author{
The indiscriminate use of Ritalin to improve academic performance \\ El uso indiscriminado de Ritalin para mejorar el rendimiento académico
}

Recebido: 08/11/2021 | Revisado: 16/11/2021 | Aceito: 17/11/2021 | Publicado: 28/11/2021

\author{
Giulia Cecília de Souza \\ ORCID: https://orcid.org/0000-0003-2904-5205 \\ Centro Universitário do Vale do Ipojuca, Brasil \\ E-mail: giuliacecilias@gmail.com \\ João Paulo de Melo Guedes \\ ORCID: https://orcid.org/0000-0003-2619-5080 \\ Centro Universitário do Vale do Ipojuca, Brasil \\ E-mail: joao.guedes@professores.unifavip.edu.br
}

\begin{abstract}
Resumo
O Ritalina como é mais conhecido comercialmente, é um dos principais fármacos estimuladores do sistema nervoso central, é o psicoestimulante mais consumido no mundo e sua principal prescrição é no tratamento para pacientes com TDAH. Este estudo tem o objetivo de expor através da literatura de artigos o uso do fármaco sem prescrição medica para fins não terapêutico, como é a questão do uso para aprimoramento cognitivo e até recreativo. Onde irá abordar também seu mecanismo de ação, o TDAH, efeitos adversos e interações possíveis que o medicamento pode causar. Neste trabalho propõe analisar como o uso indevido da droga pode trazer benéfico e maleficio há saúde do usuário, principalmente a saúde mental que é a mais afetada, prometendo horas de estudos e concentração prolongada, por isso é conhecida como a "droga da inteligência".
\end{abstract}

Palavras-chave: Ritalina; Metilfenidato; TDAH; Psicoestimulantes.

\begin{abstract}
Ritalin, as it is more commercially known, is one of the main drugs that stimulate the central nervous system, is the most consumed psychostimulant in the world and its main through the literature of articles the issue of use for cognitive improvement and even recreational. Where it will also address its mechanism of action, ADHD, adverse effects and possible interactions that the drug can cause. This paper proposes to analyze how drug misuse can bring benefits and harm to the user's health, especially mental health, which is the most affected, promising hours of study and prolonged concentration, which misuse can bring benefits and harm to the user's health, especially mental health. which is the most affected, promising hours of study and prolonged concentration, wich is why it is known as the "intelligence drug".
\end{abstract}

Keywords: Ritalin; Methylphenidate; ADHD; Psychostimulants.

\begin{abstract}
Resumen
Ritalin, como se le conoce más comercialmente, es uno de los principales fármacos que estimulan el sistema nervioso central, es el psicoestimulante más consumido en el mundo y su principal prescripción es para el tratamiento de pacientes con TDAH. Este estudio tiene como objetivo exponer a través de la literatura de artículos el uso del fármaco sin prescripción médica con fines no terapéuticos, como es el tema del uso para la mejora cognitiva e incluso recreativa. Donde también se abordará su mecanismo de acción, el TDAH, los efectos adversos y las posibles interacciones que puede ocasionar el fármaco. Este trabajo propone analizar cómo el abuso de drogas puede traer beneficios y daños a la salud del usuario, especialmente la salud mental, que es la más afectada, prometedoras horas de estudio y concentración prolongada, por lo que se la conoce como la "droga de la inteligencia".
\end{abstract}

Palabras clave: Ritalin; Metilfenidato; TDAH; Psicoestimulante.

\section{Introdução}

$\mathrm{Na}$ década de 50 a medicina, em especial a psiquiatria, expandiu seus conhecimentos em neurociência, dando então espaço aos psicofármacos para tratamentos neurológicos, atendendo então as exigências de rapidez e eficácia. Ao longo dos séculos passados a psiquiatria era uma área bastante desvalorizada, pois não ofertava métodos eficazes em diagnósticos e tratamentos, a partir da década 50, como foi mencionado anteriormente, a psiquiatria teve um avanço muito grande, podendo 
citar que após esse passo a psiquiatria vem se inovando a cada ano, pois descobre-se novos tratamentos e meios de diagnósticos para doenças desconhecidas ou pouco estudadas (A expansão do consumo da ritalina).

Em meados de 1955 foi lançada no mercado o medicamento com o nome comercial de Ritalina, com uma série de indicações, as mais estudadas no tempo era para crianças hiperativas e pacientes com casos de Narcolepsia. Com a chegada de uma nova droga do mercado e com a fama de melhorar o desempenho cognitivo e sua atenção, estudantes e profissionais de várias as áreas começaram a se interessar pelo medicamento "milagroso", mesmo que não tivesse o diagnóstico positivo para o TDAH ou qualquer outro diagnóstico neurológico que tivesse como tratamento o Ritalina.

O Transtorno de déficit de atenção (TDAH) começou a ser utilizado em 1980 pela Academia Americana de Psiquiatria como um novo conceito para agregar todos os outros distúrbios relacionados a falta de atenção e a hiperatividade.

A Ritalina é um fármaco psicoestimulador, da família das anfetaminas, muito indicada no tratamento para crianças diagnosticadas com TDAH, esse fármaco ele trabalha no sistema nervoso central (SNC) proporcionando ao usuário um controle sobre seu corpo, conseguindo se manter calmo, executar atividades sem interrupções, prestar atenção em algo importante, dentre outros inúmeros fatores.

Mas devido a fama que o fármaco ganhou houve uma procura gigantesca e hoje isso preocupa os responsáveis da saúde, pois por se tratar de um medicamento que pode causar vícios também há um uso exagerado, fazendo o Brasil chegar a $\operatorname{ser} 2^{\circ}$ maior consumido de Metilfenidato do mundo, perdendo apenas para os EUA.

Sendo assim, nós nos importamos de nos aprofundarmos mais sobre as informações sobre o fármaco e levarmos para a população, para que seja atingido o máximo de pessoas possíveis, com o intuito de deixarmos a maioria orientada sobre o fármaco, seus riscos e a real função do medicamento. Importante também enfatizar o papel do farmacêutico na orientação quando a uso irracional do medicamento.

\section{Metodologia}

Este trabalho de conclusão de curso apresenta uma metodologia qualitativa, baseada numa revisão de literatura. Tratase de uma revisão narrativa de literatura sobre o uso indiscriminado do Ritalina para o melhoramento acadêmico. Temos o intuito de avaliar através de artigos e estudos sobre a capacidade do medicamento no melhoramento intelectual em pessoas saudáveis e também alertar sobre os possíveis riscos que possa causar a saúde do usuário. Sempre buscando a forma mais clara para que todos possam se interessar e se aprofundar nos assuntos relacionados.

A revisão de literatura tem o objetivo de resumir toda a informação sobre o assunto, com um olhar crítico e amplo sobre as publicações analisadas, buscamos muitos assuntos que envolvem o tema da nossa revisão literária, assuntos como: O mecanismo de ação do Ritalina, artigos que aprofundam sobre o TDAH, entre vários outros. O presente estudo sobre o assunto foi abordado através das bases de dados: Scielo, Google acadêmico, PubMed e ANVISA. A finalidade é que o pesquisador tenha um contato maior com o assunto e passe adiante tudo que foi visto e aprendido.

\section{Revisão de Literatura}

Na década de 50 a medicina, em especial a psiquiatria, expandiu seus conhecimentos em neurociência, dando então espaço aos psicofármacos para tratamentos neurológicos, atendendo então as exigências de rapidez e eficácia. Ao longo dos séculos passados a psiquiatria era uma área bastante desvalorizada, pois não ofertava métodos eficazes em diagnósticos e tratamentos, a partir da década 50, como foi mencionado anteriormente, a psiquiatria teve um avanço muito grande, podendo citar que dai em diante a psiquiatria vem se inovando a cada ano que passa, pois descobre-se novos tratamentos e meios de diagnósticos para doenças desconhecidas ou pouco estudadas. (A expansão do consumo da ritalina). 
Em meados de 1955 foi lançada no mercado o medicamento com o nome comercial de Ritalina, com uma série de indicações, as mais estudadas no tempo era para crianças hiperativas e pacientes com casos de Narcolepsia. Com a chegada de uma nova droga do mercado e com a fama de melhorar o desempenho cognitivo e sua atenção, estudantes e profissionais de várias as áreas começaram a se interessar pelo medicamento "milagroso", mesmo que não tivesse o diagnóstico positivo para o TDAH ou qualquer outro diagnóstico neurológico que tivesse como tratamento o Ritalina.

Devido novos avanços na medicina, foi aprofundado estudos sobre o TDAH e consequentemente houve um aumento de novos casos, principalmente em crianças em fase escolar, aquelas que eram entendidas como crianças agitadas. Sendo necessária uma observação maior pelos pais e professores sobre o comportamento, pois como trata-se de um diagnóstico clínico é importante observar o cotidiano do paciente, estando em casa ou na escola, fazendo atividades simples do dia a dia, isso será uma parte crucial para o diagnóstico adequado. Deve também ser complementado por avaliações médicas. (Brian, Stephen. 2020)

O Metilfenidato é receitado frequentemente para tratamento de pacientes com o transtorno de déficit de atenção e hiperatividade, pacientes esses que poderão ser crianças ou adultos, o medicamento é indicado para crianças acima de 6 anos e deverá começar com a dosagem mínima possível. (Ramirez, Gonzalo. 2021). Atualmente considerado um medicamento sob controle especial pela Portaria 344/984, incluso na lista de SPAs "A3", sujeita a notificação da receita "A", com estrutura química semelhante há anfetaminas.

A comercialização foi aprovada no Brasil em 1998 e desde então a demanda vem crescendo ao longo dos anos, preocupando responsáveis por uso abusivo do medicamento. O Brasil é o $2^{\circ}$ país que mais consome o Metilfenidato em todo o mundo, ficando atrás somente dos EUA. A ANVISA divulgou que em 2011 foram vendidos mais de $413 \mathrm{Kg}$ de Metilfenidato (ANVISA, 2012).

O Ritalina pode causar dependências ao usuário quando usada de forma ilícita e sem acompanhamento médico, essa substância é da classe das anfetaminas, classe essa que tem uma grande chance de causar vício, podendo causar abstinência após a pausa ao o uso, como perca de sono, tremores, alucinações, dentre outros. (Bacelar, Anna Beatriz. 2018)

Esse medicamento é muito conhecimento no meio acadêmico como "a pílula da inteligência" ou "a pílula milagrosa", pois teria ela como um medicamento que aumenta a atenção e a cognição, fazendo com quem estudantes tenham notas satisfatórias em provas e concursos. (Bachi, Eleonora. 2016). Embora tem estudos que comprovam que a droga não faz efeito em usuários que não tem nenhum dos quadros neurológicos apresentado anteriormente. (Bacelar, Anna Beatriz. 2018)

\subsection{Mecanismo de ação}

O medicamento tem como sua principal composição o metilfenidato, que é um forte estimulador do sistema nervoso central (SNC), ele age ativando algumas partes do cérebro quando não estão funcionando devidamente, inibindo então a recaptação da dopamina e noradrenalina, fazendo com o que aumente os níveis dos mesmos no SNC, consequentemente melhorando suas emoções e sua concentração. O fármaco impede que as catecolaminas sejam recapturadas pelas as terminações nervosas, aumentando o tempo de ativação dos neurotransmissores (Pastura, Giuseppe. 2004).

Há estudo que alegam que seu mecanismo de ação não está completamente compreendido, mas que a ativação por meio do medicamento acontece quando está havendo alguma falha na liberação natural dos neurotransmissores (Coelho, Vinicius. 2019).

Estudos também mostraram que o medicamento não funciona em pacientes saudáveis que fazem o uso da droga de modo indevido, com o intuito de melhorar seu desempenho acadêmico. Apesar que muitos usuários alegarem que a pílula cumpre com todos os "benefícios" prometidos e conseguem resultados satisfatórios com o uso da mesma, embora não se sabe se realmente pode acontecer ou seja um processo psicológico (Pasquini, 2013). 


\subsection{Transtorno do déficit de atenção com hiperatividade (TDAH)}

Os argumentos sobre o aumento do Ritalina nos últimos anos é que o TDAH cresceu de uma forma assombrosa, devido estudos recentes e mais informações para a população, com isso foram descobertas mais pessoas que tem o transtorno e é esperado que continue aumentando

O TDAH como é mais conhecido, é um transtorno de déficit de atenção e hiperatividade, que acomete o sistema nervoso central, onde acontece um desequilíbrio neurológico. Temos cerca de 100 bilhões de neurônios que se conectam entre si e assim formam redes complexas para que executemos ações, as informações passam pelo meio dos neurônios e essa ação é conhecida de sinapse, isso acontece com ajuda de substâncias químicas chamadas de neurotransmissores, dopamina e noradrenalina, que são tipo exemplo de mensageiros que levam as informações para o corpo. Pessoas com TDAH não desempenham esse processo adequadamente em algumas regiões do cérebro, em especial no lobo frontal que é a região do cérebro responsável pela central de comandos do nosso corpo, responsável pela execução de tarefas, administração de tempo, controle de impulsos, dentre outros.

Ainda não se sabe o real motivo que desencadeia o TDAH, mas apresenta forte componente genético, em 50\% dos casos o pai ou a mãe tem, uma grande parte dos pais só descobre que tem quando procura ajuda para o filho e vai notando semelhança nos sintomas. No Brasil 5,9 milhões de adultos tem o transtorno e 5,3\% das crianças, acomete 1 a cada 20 crianças.

Geralmente crianças são diagnosticadas em fase escolar, pois com o acompanhamento e observação dos professores pode-se haver uma comparação de comportamento com outras crianças, observando sempre os sinais que a criança apresenta, como não se manter por muito tempo sentado, mexer as pernas repetidas vezes, mudar de assunto com frequência, impulsividade, entre outros. Com esses sinais é importante que os pais procurem um especialista para que se tenha um diagnóstico adequado e faça o tratamento certo, especialistas que deve ser procurado são neurologistas, psiquiatra e em casos de crianças é importante de um acompanhamento de um neuropediatra.

Crianças com TDAH muitas vezes sofrem bulling na escola devido seu comportamento e isso acaba agravando seu quadro e desenvolvendo outros problemas, como uma depressão ou ansiedade, como também prejudica o desempenho escolar, por receio de tirar alguma dúvida e sofrer com os colegas.

Adultos com o transtorno e que não fazem o tratamento também tem dificuldades no seu dia a dia, tem mais chances para estarem desempregados, não prosseguir com um relacionamento ou de acontecer um acidente. Muitos dos casos o adulto só descobre seu problema por causa de outros, como uma ansiedade, depressão ou uso excessivo de álcool, com a visita ao médico ele se aprofundará no histórico do indivíduo e dará seu diagnostico, assim começando o tratamento.

Alguns critérios usados no diagnóstico é a frequência e intensidade que as coisas acontecem, como por exemplo o esquecimento repetido de coisas do dia a dia. É importante também que seja colhido relatos do paciente, pais e escola, sempre procurando saber se ele ouve bem, enxerga bem e dorme bem, porque são fatores que são prejudicados pelo TDAH e que não seja esquecido nada na hora da avaliação, porque quaisquer o sintoma omitido pode dar um diagnostico incorreto.

$\mathrm{O}$ tratamento divide-se em 3 partes, a psicoeducação, onde irá vir a orientação aos pais e ao próprio paciente, a psicoterapia que é conhecida como TCC (Terapia cognitiva e comportamental), irá trabalhar sua atenção e seu comportamento com um especialista e a medicação, que irá ser prescrita a partir de 6 anos, onde médico irá avaliar se tem a necessidade ou não. Os medicamentos são prescritos sempre os de $1^{\mathrm{a}}$ linha, como é o caso do Ritalina, um psicoestimulador e é muito importante o acompanhamento regular ao médico para que ele avalie o medicamento e faça experiências de interrupção para avaliar se precisa manter. O medicamento pode prejudicar o crescimento em crianças, por isso pede-se que em tempos de férias seja dado uma pausa. 
O paciente deve-se fazer o que ele gosta em certos tempos do dia, por exemplo um exercício físico, pois quando as atividades dão prazer ao indivíduo estimula uma área do cérebro que chama de centro de recompensa, que aumenta a produção da substância química, a dopamina, essa carga ajuda o lobo frontal a aguçar o foco e dar mais foco.

Após pesquisar descobriu que o TDAH não tem cura, acompanha o indivíduo para o resto da vida, em $90 \%$ dos casos, mas pode haver regressão ou aumentar o grau dependendo do tratamento feito.

\subsection{A influência do Ritalina entre acadêmicos}

Uma questão bastante importante vem preocupando especialistas na área da saúde, o uso exorbitante da Ritalina no meio acadêmico, uma pílula medicamentosa que promete uma inteligência e concentração a mais nos estudos e avaliações. Esse medicamento é muito usado no melhoramento cognitivo por pacientes com TDAH e com a notícia que o metilfenidato melhorava a cognição e que os pacientes obtinham sucesso em suas tarefas, estudantes e trabalhadores começaram a se interessar pelo mesmo, com o intuito de melhorar sua concentração, evitar o cansaço e o estresse mental, assim lhe dando mais disposição para concluir suas tarefas (Bacelar, Anna Beatriz. 2018).

Usuários afirmam que quando fazem o uso o desempenho aumenta bastante e conseguem um avanço em suas atividades. Embora não há estudos que afirmem que o medicamento faça efeito adequado em usuário que não apresentam transtornos neurológicos e com isso a preocupação de especialistas aumentam, devido a fama do medicamento se espalhar de uma forma positiva e a fácil acesso através da internet para a compra.

Esse medicamento faz parte da classe das anfetaminas, classe essa que pode desenvolver vício e dependência e por isso que esse medicamento precisa de controle especial e de acompanhamento especializado (ANVISA, 2013). A procura na maioria das vezes é por estudantes ou pessoas que estão prestes a passar por um concurso, onde buscam um suporte para se aprofundar nos estudos, podendo ficarem por mais tempo acordados sem perder o foco, uma concentração maior do que a de costume, dentre outros fatores que o estudante espera da droga. Vemos também a procura por empresários ou trabalhadores que trabalham em uma carga de estresse grande. (Portugal, Siqueira. 2011)

O Metilfenidato hoje está sendo um refúgio para aqueles que querem obter sucesso o mais rápido possível, devido a grande procura por sempre estarem se saindo bem, como também o mercado de trabalho mais exigente e concursos mais concorridos. Fazendo com que estudantes procurem um meio mais fácil e rápido para obterem resultados positivos e não passarem por frustações posteriores. Porém muitos desses que procuram o medicamento, não sabem e não procuram saber os riscos que pode acontecer a sua saúde, pois como trata-se de um medicamento de prescrição especial, é importante que seja avaliado por um médico e que tenha o acompanhamento adequado, pois ele pode causar dependência, falta de apetite, alucinações, dentre outros. (Costa, Vinicius Coelho, 2019). Também podemos citar o uso do mesmo por mulheres na sua maioria para a perca de peso, pois ele tira o apetite. (Tsuda e Christoff, 2015).

O Ritalina é adquirido muitas vezes através de sites nacionais e internacionais de modo ilegal ou por terceiros que fazem o uso do mesmo, mas podemos ver também pessoas que procuram um médico e forjam sintomas que pessoas com TDAH tem, como falta de atenção, inquietude, desobediência e etc; para que consigam a receita necessária. (Barros, Ortega. 2011)

Em 2013 foi feito um Alerta pela ANVISA do número crescente de usuário do medicamento em todo o país, um crescimento cerca de 164\% no consumo, entre 2009 a 2011. (ANVISA, 2013). Algumas pesquisas mostraram o percentual dos estudantes, em especial da área da saúde, que não possuem o transtorno e utilizaram o medicamento varia de 3 a 35,5\% (LOW, Gendaszek, 2002).

É crescente o número de pessoas que estão adotando esse novo "método" de estudos em todo o mundo, assim as vendas excederam 1 bilhão de dólares em 2015 e vem crescendo a cada ano. (Chintapalli, 2015). A facilidade aumenta devido 
existirem sites nacionais e internacionais que fazem a venda sem prescrições e uma divulgação ampla em sites e blogs, enaltecendo as ações farmacológicas do Ritalina.

Usuários usam meios na internet mais restritos para compartilharem experiências e auxiliarem os demais, como o medicamento adequado para tal função procurada, e é passada também dosagem adequadas, combinações medicamentosas, entre outras. É um meio de comunicação entre usuários ou pretendes, onde trocam ideias, acontecem relatos diários. (Maia, Fidelis, 2018)

A cafeína e o guaraná são psicoestimulantes mais utilizados entre os acadêmicos, devido melhorarem o humor e diminuírem o cansaço físico e mental (Mendonça, 2002), podemos citar energéticos que em sua composição apresentem Taurina. É indicado também o uso do medicamento Ginko Biloba, que aumenta o fluxo sanguíneo no cérebro. Todos citados acima podem substituir e tem uma ação semelhante ao Metilfenidato, sem citar que causa riscos menores a saúde. (Menezes, Nomerg, Lenzi, 2017).

Com todas essas alternativas acima, o Ritalina ainda é o mais procurado e o mais falado em toda a mídia e por ser uma substância nova, geralmente nunca usada antes por estudantes, no início o organismo age bem a ela e faz com o que a notícia da "pílula da inteligência" se espalhe muito mais rápido e com boas informações.

Estudos apontam que o uso em pessoas sem TDAH que fazem o uso do medicamento, pode prejudicar seu desempenho nas atividades, pois pode acontecer o efeito inverso. Prejudicando a realização das atividades, capacidade de planejamento, cognição, entre outros. (Maia, 2017).

O mundo está passando por um período de expansão e inovações em todos os setores, buscando sempre os melhores para ocuparem as áreas propostas, sendo assim os cargos ficam mais concorridos e mais dificultosos, fazendo com que os interessados deem o seu melhor, porém as vezes não é o suficiente para que se consiga e com isso é onde procuram novos meios. É onde entra o uso indevido de substâncias, como o Metilfenidato, estão há procura de algo que possa melhorar seu desempenho e são apresentados há novas experiências, com a fama que o medicamento é o milagroso dos estudos o indivíduo se interessa logo e começa a fazer uso mesmo que não saiba os riscos que pode ocasionar a sua saúde.

Cada vez mais é entendido como normal fazerem o uso de medicamentos para o aprimoramento intelectual, como o intuito de uma mudança neurológica, mantendo uma carga horaria de estudo mais extensa, gestão de atenção e produtividade maior, eficiência ao receber as informações e ter um controle maior em si mesmo. Mas acaba que tem pessoas, em sua minoria, que alegam que acabam ficando reféns desse medicamento, pois só conseguem a concentração almejada quando fazem o uso da droga.

Não é com frequência que vemos falando sobre, mas acontece bastante em festas a mistura do medicamento com bebidas alcóolicas para fins recreativos, porém o uso de aprimoramento cognitivo e o uso recreativo são ambos ilegais e os usuários de ambos estão vulneráveis a desenvolverem dependência química. (Itabohary, Claudia, 2009).

\subsection{Efeitos adversos}

Existe uma grande possibilidade de pessoas desencadearem dependência quando usado de forma ilegal ou sem consentimento médico, devido aos fatores de risco, podendo alterar sua emoção, consciência, bem estar. O metilfenidato age no SNC alterando a percepção e podendo causar alucinações devido seu mecanismo de ação no cérebro, sua atividade é bem parecida com drogas que são consideradas proibidas, como ectasy e cocaína. (Cordeiro, 2017)

O Metilfenidato pode causar efeitos colaterais como cefaleia, sudorese, falta de apetite, insônia, ansiedade e irritabilidade, pessoas também podem apresentar quadros de abstinência, geralmente quando fazer o uso por um período prolongado. 
Com o uso abusivo pode desencadear alterações cardíacas, como taquicardia, arritmias e angina, no SNC também, como agressividade, mudança de humor, confusões, dores de cabeça, entre outros, no sistema gastrointestinal pode ocasionar dores abdominais, perda de apetite, náuseas. Nos casos mais extremos podemos ver alucinações, anorexia, euforia e convulsões. (Silva ÍHA, Leite AHP, Telles AMS, 2020)

Muitos dos usuários fazem o uso sem procurar se informar sobre as possíveis ações adversas que pode ocasionar, dando ouvidos apenas para os possíveis benefícios que ele pode proporcionar, podendo agravar alguma patologia existente.

\subsection{Contraindicações}

- Gestantes

Não deve ser utilizado por gestantes, pois não há estudos suficientes que garantam a segurança da mulher e do bebê, trata-se de um medicamento de categoria $\mathrm{C}$ de risco a gestantes.

Os médicos devem avaliar se o benefício do uso supera o risco e não deve ser usado sem o consentimento do médico.

\section{- Lactação}

O metilfenidato foi excretado no leite materno, atingindo uma razão de leite/plasma de 2,5. O médico em conjunto com a mãe devem tomar uma decisão, levando em conta qual terá um benefício maior, a amamentação para o bebê ou o tratamento da mãe.

- Pacientes pediátricos abaixo de 6 anos

A Ritalina não deve ser utilizada em pacientes abaixo de 6 anos pois não foram estabelecidos os paramentos de segurança e eficácia nessa faixa etária.

\section{- Condições Cardiovasculares}

Pacientes com anormalidades estruturais cardíacas não devem fazer o uso, pois o medicamento pode elevar o risco de morte súbita devido aos efeitos simpatomiméticos de um fármaco estimulante. Antes de começar o tratamento o paciente deve fazer exames cardíacos para tirar quaisquer dúvidas.

Pacientes com hipertensão grave também não são indicados fazerem o uso, pois o Metilfenidato aumenta os batimentos cardíacos e o fluxo sanguíneo no coração. Caso o paciente não tenha uma $2^{\mathrm{a}}$ opção e tenha que fazer o tratamento com o fármaco, deve ser monitorado em intervalos por um cardiologista. Se os sintomas aparecerem após o início do tratamento deve ser submetido imediato a uma bateria de exames.

\section{- Condições Cerebrovasculares}

Pacientes com anormalidade cerebrais, como por exemplo, aneurisma cerebral não deve ser tratado com o Ritalina, já pacientes com fatores de risco adicionais devem ser avaliados regularmente.

\section{- Condições Psiquiátricos}

O fármaco Ritalina não deve ser usado por pacientes com psicoses agudas, manias agudas ou tendência suicida aguda, antes de iniciar o tratamento os pacientes devem ser avaliados quanto ao distúrbio psiquiátrico e ao histórico familiar. Em casos de sintomas psiquiátricos emergentes ou exacerbação dos sintomas o médico deve levar em questão se o benefício supera o potencial de risco.

Quando o paciente apresenta sintomas psicóticos, como alucinações, deve fazer a interrupção do medicamento, em casos de comportamentos agressivos deve ser avaliado pelo médico a dosagem ou até ser considerado a descontinuação do tratamento. Pacientes com tendências suicidas devem ter um monitoramento constante e isso deve ser passado ao próprio 
paciente e aos seus cuidadores, qualquer que seja as mudanças de comportamento deve ser imediatamente avisado ao médico. A aparição de tiques em usuários do medicamento é comum e deve ser observado e investigado pelo médico antes do início do tratamento, pacientes com histórico de síndrome de Tourette não deve fazer o uso.

\section{- Priapismo}

Geralmente é notificado que após o início do tratamento é comum ereções prolongadas e dolorosas, pacientes que desenvolverem devem procurar imediatamente atendimento médico. O priapismo se desenvolve geralmente quando se faz um uso prolongado do fármaco e há uma pausa devido a retirada.

\section{- Retardo do Crescimento}

Quando o medicamento é usado por crianças é relatado uma diminuição no ganho de peso e um leve retardo no crescimento. O desenvolvimento da criança deve ser monitorado, caso haja um retardo grave o tratamento deve ser suspenso.

\section{- Convulsões}

O uso do Ritalina em pacientes com epilepsia deve ser feito com cautela e monitorado constantemente, pois o medicamento pode causar o aumento na frequência das crises.

- Abuso de medicamentos e dependência

É recomendado cautela em pacientes instáveis ou com histórico de dependência química pois pode induzir uma dependência psicológica.

- Descontinuação

É necessário supervisão durante a retirada do medicamento, pois pode ocorrer uma depressão ou ansiedade por falta do medicamento, as vezes é preciso um acompanhamento há longo prazo.

- Pacientes pediátricos abaixo de 6 anos

A Ritalina não deve ser utilizada em pacientes abaixo de 6 anos pois não foram estabelecidos os paramentos de segurança e eficácia nessa faixa etária.

\subsection{Interações medicamentosas}

- Medicamentos anti-hipertensivos

A Ritalina pode diminuir a efetividade do medicamento utilizado para o tratamento da hipertensão, pode também elevar a pressão sanguínea.

\section{- Bebidas Alcóolicas}

Não é indicado o uso do medicamento junto ao álcool, pois o álcool exacerba os efeitos do medicamento no SNC, podendo deixar o paciente agressivo e até fora de si.

- Medicamentos dopaminérgicos

Não é indicado pois os medicamentos iriam fazer mecanismo de ação contrário, podendo agravar alguma das patologias.

\subsection{O papel do farmacêutico}

A medicalização nos tempos atuais está muito grave, principalmente o fato da automedicação está sem controle, sendo assim, o papel do farmacêutico é de extrema importância na orientação sobre o uso desenfreado dos medicamentos. Sabe-se 
que o uso racional dos medicamentos é um assunto bem trabalhado ultimamente, mas infelizmente não é toda a população que segue o que as áreas competentes tentam trabalhar a fundo, pode ser por deficiência de informações ou por uma cultura que foi construída ao longo dos anos. (Firmino, 2012)

Como é de papel do farmacêutico sempre procurar promover a saúde do paciente e ser constatar algo incorreto deve intervir no uso do medicamento, procurando sendo a promoção da saúde do paciente. Sempre deixando claro os riscos que o medicamento pode causar, mesmo que seja prescrito por um médico, alertando sobre interações medicamentos e efeitos colaterais, sendo instruindo sempre ao melhor. É de extrema importância também o farmacêutico sempre buscar limitar o uso ilegal de medicamentos psicotrópicos, visando uma diminuição no uso abusivo.

\section{Resultados e Discussão}

Os resultados encontrados foram obtidos através de artigos, ao todo foram analisados 32 artigos que tem relação com o tema escolhido da pesquisa, foram incluídos sobre o TDAH e o uso indevido de psicoestimuladores com fins no melhoramento acadêmico. Foi observado também a opinião dos escritores sobre se o fármaco faz efeito adequado em pacientes saudáveis, em sua maioria foi mostrado que o fármaco só age de forma correta em pacientes com o distúrbio neurológico, (Chaterje, 2009) mas podemos ver citações de apoiadores do uso do fármaco para o melhoramento cognitivo.

Em sua grande maioria o uso ilegal do medicamento é procurado por jovens, em idade escolar ou universitária, pois são pressionados para terem um desempenho exemplar e alcançar objetivos. Na vida adulta também podemos ver a grande procura, pois o mercado de trabalho hoje está muito competitivo e exige o melhor de seus colaboradores. (Sahakian, 2009).

As drogas com ações psicoestimuladores são as mais procuradas por aqueles que querem ampliar a sua capacidade intelectual, aumentar a sua carga horaria de estudos ou chegarem no seu objetivo, seja ele uma prova, um concurso, um emprego. As drogas são substâncias que tem a capacidade de aumentar o estado de alerta e concentração do usuário. (Gonçalves Cs e Pedro Rmr, 2018).

Existem poucos estudos sobre o mecanismo de ação do Ritalina em pacientes saudáveis e isso vem preocupando os responsáveis, pois vem aumentando o índice de uso e isso gera uma incerteza pois não se sabe ao certo o mecanismo do fármaco (Faraone, 2010) Também preocupa pelo fato de causar dependência aos usuários, por se tratar da classe das anfetaminas, o uso sem prescrição médica e o fácil acesso ao medicamento através da internet.

\section{Conclusão}

Ao longo desse trabalho nosso objetivo foi deixar o mais explicito possível os fatores que o Metilfenidato pode causar aos usuários, através de artigos que abordassem o tema. Nossa intenção foi mostrar para os leitores e interessados no medicamento que o uso indiscriminado e sem acompanhamento médico pode causar sérios riscos há saúde. Conseguimos bons artigos que passaram o esperado e facilitou muito a nossa perspectiva quanto aos artigos encontrados.

A internet fornece várias informações, porém de forma incompleta, não deixa claro a qualidade do tratamento, a duração, o mecanismo de ação, dentre outros fatores. $\mathrm{O}$ individuo que busca pelo uso do medicamento sem consentimento médico está colocando sua saúde em risco, pois não se sabe os efeitos do medicamento em pacientes saudáveis, não sabe os possíveis efeitos adversos que ele pode causar.

Então concluímos que é importante que tenha mais estudos sobre o uso em pacientes saudáveis, pois não vemos o suficiente para podermos ter uma visão ampla do uso em curto ou longo prazo, procurar também sobre a finalidade do medicamento e sempre com uma orientação médica, pois medicamento não é uma coisa simples, tem muito o que ser entendido e aprofundado. 


\section{Referência}

ANVISA. (2014). Metilfenidato no tratamento de crianças com transtorno de déficit de atenção e hiperatividade. BRATS: boletim brasileiro de avaliação de tecnologia em saúde. Ano, VIII N²3, p 1-18

Silva, A. H. I., Leite, P. H. A. \& Telles, S.M.A. (2020). Uso indevido de Metilfenidato por universitários da área da saúde: revisão sistemática. universidade federal de pernambuco - centro de ciências da saúde. $\mathrm{v} 31, \mathrm{n}^{\circ} 4, \mathrm{p} 33-42$.

Bacelar, Anna Beatriz. (2018). Ritalina, uma droga que ameaça a inteligência. REVISTA DE MEDICINA E SAÚDE DE BRASILIA. BRASILIA. p 99-112

Barros, Denise \& ORTEGA, Francisco. (2011). Metilfenidato e aprimoramento cognitivo farmacológico: representações sociais de universitários. saúde e sociedade, v. $20 . \mathrm{N}^{\mathrm{o}} 2$, p. $350-362$.

Batista, K.L., Batista, C.E., FErreira, F.D., Matos, P.H., Magalhães, M.D. \& Oliveira, C.M.L.M. (2018). O uso da Ritalina em crianças com TDAH, uma revisão teórica. HUMANAE. v $12, \mathrm{n}^{\circ} 2, \mathrm{p} 2-18$.

Bessa, M. A. et al., (2012)., Abuso e dependência de anfetamínicos. Projeto Diretrizes. São Paulo. p 3-15

Bicalho G.P.P., Bohre T.C.L. \& Decotelli M.K. (2013). A droga da obediência: medicalização, infância e biopoder: notas sobre clínica e política. Rio de Janeiro - RJ. universidade federal do rio de janeiro. $\mathrm{v} 33$, ano 2, $\mathrm{p} 446-459$.

Caliman V.L. \& DomitroviC Nathalia., (2017). Geração Ritalina e a otimização da atenção: notas preliminares. centro de estudos sociais laboratório associado. $\mathrm{n}^{\mathrm{o}} 439, \mathrm{p} 1-10$.

Coelho, Vinicius., (2019)., Uso indiscriminado do metilfenidato para o melhoramento acadêmico: Uma revisão de literatura. FACULDADE MARIA MILZA. Governador Mangabeira-Bahia. p 13-37.

Conceição, P.A., Freitas, Q.B., Delmondes, P.H. \& Borges, S.A.M. (2019). Uso do Ritalina no melhoramento acadêmico nos cursos de Enfermagem e Farmácia. revista eletrônica interdisciplinar de barra de garças. Barra de Garças - MT. v 11, nº 1 , p 124-131.

Cordeiro, Nicolas \& Pinto Caetano Moreira Rodrigo. (2017). Consumo de estimulantes cerebrais em acadêmicos da área da saúde na cidade de Ponta GrossaMT. biblioteca digital de periódicos. Ponta Grossa - MT. v 18, $\mathrm{n}^{\circ} 2, \mathrm{p} 23-38$.

Clark, Luke., Chamberlain, R. S. \& Sahakian, J. B. (2009). Mecanismos neurocognitivos na depressão: implicações para o tratamento. REVISÃO ANUAL DE NEUROCIENCIA. v 32, p 57-74.

Faraone, S. V. (2010). Genética molecular do transtorno de déficit de atenção e hiperatividade. clinica de psiquiatria norte americana. v 33, ${ }^{\circ} 1$, $\mathrm{p} 159-180$.

Filho, P.J., Rodrigues, R.A., Leão, A.M., Sousa, P.J., Araújo, M.J., Lima, G.E., Costa, D.D., Freitas, A.A., Cardoso, R.M. \& Carvalho, B.R.L. (2020). Medicação de alto risco: reflexão da Ritalina. Teresina- PI. revista eletrônica acervo saúde. v 12, nº $12, \mathrm{p} 2-8$.

Gonçalves, C.S. \& Pedro, R.M.R. (2018). Drogas da inteligência cartografando as controvérsias do consumo de ritalina para o aprimoramento cognitivo.Psicología, Conocimiento y Sociedad. Trabajos originales. v 8, nº 2, p 71-94.

Itaborahy, Claudia. (2009). A Ritalina no Brasil: Uma década de produção, divulgação e consumo. BIblioteca digital de teses e dissertações. Rio de Janeiro. p 10-32

João, O., Jordi, A., Philip, S.W., Ronald, C. K., Sergio, A.G., Sing, L., Somnath, C. \& Ustun, B.T. (2009). A carga global dos transtornos mentais: uma atualização das pesquisas de saúde mental mundial da OMS. psychiatric sciences. v 18, nº 1, p 23-33.

Junqueira, Livia., Itaborahy, Claudia \& Ortega, Francisco. (2010). A Ritalina no Brasil: produções, discursos e práticas. departamento de políticas e instituições em saúde. Rio de Janeiro - RJ. v 14, nº31, p 399-510.

Lenzi, R. V., Nomerg, O. K. \& Menezes, S. S. A. (2017). O uso de psicoestimulantes por acadêmicos de uma instituição de ensino superior do Estado de Rondônia. repositorio intitucional. p 1-13.

Luzio, A.C., Santos, P.Y.K., Dionisio, H.G., Silva, P.C.A. \& Yasui, Silvio. (2012). A explosão do consumo da Ritalina. revista de psicologia da UNESP. Campinas - SP. v 11, $\mathrm{n}^{\circ} 2$, p 2-14.

Maia, Fidelis. (2018). As "DROGAS DA INTELIGÊNCIA": apropriações e subjetividade no uso de psicofármacos para potencializar o desempenho cognitivo. reunião brasileira de antropologia.

NOvartis., (2021)., Bula do medicamento Ritalina. São Paulo. novartis biociências S.A.

Santana, Ana Paula \& Signor Rita. (2016). TDAH e medicalização. plexus editora. São Paulo - SP. p 9-15.

Silva, A. H. I., Leite, P. H. A. \& Telles, S.M.A. (2020). Uso indevido de Metilfenidato por universitários da área da saúde: revisão sistemática. universidade federal de pernambuco - centro de ciências da saúde. $\mathrm{v} 31, \mathrm{n}^{\circ}$ 4, $\mathrm{p} 33-42$.

Stolf Fabiola. (2019). A influência do "modelo centrado na doença" no uso do medicamento para problemas de aprendizagem na escola. revista polítICA $e$ sociedade. v 19, $\mathrm{n}^{\circ} 46, \mathrm{p} 242-268$.

Varella, Drauzio. (2021). Tudo ao mesmo tempo. Fantástico. G1. Disponível em: https://g1.globo.com/fantastico/noticia/2021/10/04/drauzio-varella-explica-otripe-do-tratamento-para-o-tdah.ghtml 